\title{
Reliability Assessment of Solid State Lasers Based on Step Stress Accelerated Degradation Test
}

\author{
Xiaolu Fu, Jun Yang \\ School of Reliability and Systems Engineering, Beihang University, Beijing
}

Keywords: SSADT; solid state lasers; reliability assessment.

\begin{abstract}
Highly-reliable and long-lifetime solid state lasers require the extremely high reliable assemblies over a long period of time. In order to shorten the test time, we apply step stress accelerated degradation test (SSADT) to assess solid state lasers' reliability. Firstly, the assumptions and introductions of SSADT are given. Then, we establish a performance model of solid state lasers which is determined by two factors: photoelectric change efficiency and input electrical power. Next, a degradation model is set up under the assumption that the degradation of photoelectric change efficiency follows Wiener process. On the basis of failure mode effect of solid state lasers, accelerated model is determined and thus reliability model is obtained. The maximum likelihood is used to estimate the parameters of reliability model. Finally, an example of solid state lasers' SSADT is presented to implement the proposed method.
\end{abstract}

\section{Introduction}

Nowadays, solid state lasers have played an important role in many industrial fields. When assessing the reliability of highly-reliable, long-lifetime and expensive products such as solid state lasers, the traditional Accelerated Life Test (ALT) tends to take a large amount of time and money with little helpful information of the products. An alternative approachAccelerated Degradation Test (ADT) is popular for the reason that it can provide abundant and valuable reliability information efficiently ${ }^{[1]}$. Furthermore,Step Stress Accelerated Degradation Test (SSADT) is superior to Constant Stress Accelerated Degradation Test (CSADT) in the respects of sample size, test cost and time ${ }^{[2]}$.

Researches on accelerated degradation test mainly include three aspects: statistical analysis, optimization design and engineering ${ }^{[3]}$. Meeker and Escobar ${ }^{[4]}$, and $\mathrm{Chao}^{[5]}$ reviewed the degradation literature, surveyed its wide applications and described statistical methods for ADT models. Tseng and Peng introduced a stochastic diffusion process to model the product's degradation path ${ }^{[6]}$. Wang et al. dealt with efficient SSADT plans under the conditions that the rate of degradation is consistent with the inverse Gaussian process ${ }^{[7]}$. This paper put forward a SSADT method for solid state lasers to evaluate their life and reliability. First, we introduce the SSADT in testing assumptions, execution process and data structure. Next, the degradation process of solid state lasers' behaviour is described and the reliability model is obtained with models of Wiener process and Inverse Power Law. Then maximum likelihood estimation (MLE) is used to estimate the parameters of reliability model. At last, an example of solidstate lasers is proposed to illustrate the method.

\section{Step stress accelerated degradation test}

In this section, we make clear the testing assumptions and loading process of SSADT. After that, we show what the testing data looks like under SSADT.

\subsection{Testing assumptions of SSADT}

Accelerated test and the establishment of reliability models are usually based on certain assumptions. This paper proposes the following assumptions by reference to relevant researches at home and abroad ${ }^{[8-9]}$.

1) There is path and rules to follow for the degradation process of photoelectric change efficiency.

2) Under each accelerated stress level, the failure mechanism of solid state lasers is in line with that at normal-used stress level.

3) Under the input electrical power stress level $S_{k}$, the degradation process of the photoelectric change efficiency $\mathrm{Y}\left(\mathrm{t} \mid \mathrm{S}_{\mathrm{k}}\right)$ relies only on the current stress level $\mathrm{S}_{\mathrm{k}}$, and the degradation already accumulated, with no sort of relation to the way it accumulated.

\subsection{Loading process of SSADT}

To carry out a SSADT, we should first determine a set of accelerated stress levels $\mathrm{S}_{1}<\mathrm{S}_{2}<\cdots<\mathrm{S}_{\mathrm{K}}$ which are higher than normal stress level $\mathrm{S}_{0}$. At the beginning of the test, put all test samples under initial stress $S_{1}$ and record related performance degradation. Switch to the next stress $S_{2}$ when completing the test time under $\mathrm{S}_{0}$. Go on the above steps until the test is completed under the highest stress level up to $\mathrm{S}_{\mathrm{K}}$. The execution process of the test is shown in Figure 1.

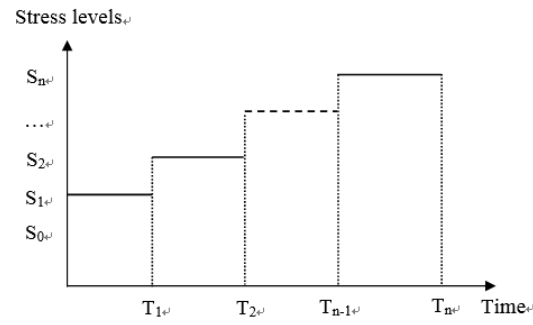

fig.1 SSADT loading process 


\subsection{Data structure of SSADT}

Suppose the sample size is $\mathrm{n}$ and the stress levels are $\mathrm{K}, \mathrm{S}_{1}<$ $\mathrm{S}_{2}<\ldots<\mathrm{S}_{\mathrm{K}}$. Performance inspection interval of lasers' output power is $\Delta \mathrm{t}$. There are $\mathrm{M}_{\mathrm{k}}$ times of performance examination in the process of SSADT, each of which is under the stress level $\mathrm{S}_{\mathrm{k}}$, besides, the accumulated inspections are $\mathrm{M}=\mathrm{M}_{1}+\mathrm{M}_{2}+\ldots+\mathrm{M}_{\mathrm{K}} . \mathrm{P}_{\mathrm{ikj}}$ is the inspection result of the $\mathrm{jth}$ inspection on the ith sample under the $S_{k}$ stress level $(j=1$, $2, \ldots \mathrm{Mk}, \mathrm{i}=1,2, \ldots \mathrm{n})$. The data structure of the test is shown in Table I.

Table I Data structure of SSADT

\begin{tabular}{|c|c|c|c|c|}
\hline \multirow{2}{*}{$\begin{array}{l}\text { Stress } \\
\text { level }\end{array}$} & \multirow{2}{*}{$\begin{array}{l}\text { Sample } \\
\text { number }\end{array}$} & \multicolumn{3}{|c|}{$\begin{array}{l}\text { Performance inspection moments } \\
\text { and results }\end{array}$} \\
\hline & & $1 \mathrm{st}$ & $\cdots$ & $\mathrm{M}_{\mathrm{k}}$ th \\
\hline \multirow{3}{*}{$\mathrm{S}_{1}$} & 1 & $P_{111}$ & $\cdots$ & $\mathrm{P}_{11 \mathrm{M} 1}$ \\
\hline & $\ldots$ & $\cdots$ & $\ldots$ & $\ldots$ \\
\hline & $\mathrm{n}$ & $P_{n 11}$ & $\cdots$ & $\mathrm{P}_{\mathrm{n} 1 \mathrm{M} 1}$ \\
\hline$\ldots$ & $\ldots$ & $\ldots$ & $\ldots$ & $\ldots$ \\
\hline \multirow{3}{*}{$\mathrm{S}_{\mathrm{K}}$} & 1 & $\mathrm{P}_{1 \mathrm{~K} 1}$ & $\ldots$ & $P_{n K M K}$ \\
\hline & $\cdots$ & & $\ldots$ & \\
\hline & $\mathrm{n}$ & $\mathrm{P}_{\mathrm{nK} 1}$ & $\cdots$ & $\mathrm{P}_{\mathrm{nKMK}}$ \\
\hline
\end{tabular}

\section{Model building}

Before using the accelerated degradation test to evaluate the reliability of the product, we should first establish the mathematical relationship between the life and stress of the product, that is, the acceleration model. So that we could extrapolate the product' reliability under normal working stress from the test data in the harsh stress levels.

\subsection{Solid state lasers' output power model}

Generally, solid-state lasers' optical output power is related to two characteristics: input electrical power and photoelectric change efficiency. When conducting a SSADT for solid-state lasers, the input electrical power remains unchanged until it switches to a higher level, but the photoelectric change efficiency degrades all the time. We assume that the optical output power $(\mathrm{P})$ is equal to photoelectric change efficiency (Y) times input electrical power $(\mathrm{S})$ :

$$
P=Y \cdot S
$$

\subsection{Degradation process model}

Since the information obtained in the accelerated degradation test is not the product' failure data, but the degradation of the product' performance under stress and time, it is necessary to establish a reliability model based on the product' degradation process. Due to factors such as unit-to-unit variability and external noise, the degradation of the product is a stochastic process and the determined degradation functions are not suitable here. So in this study, we apply drift Wiener process to describe the degradation process of photoelectric change efficiency.

$$
Y(t)=y_{0}-d(s) \cdot t+\sigma B(t)
$$

Where, $y_{0}$ is the initial degradation value before the test; $\mathrm{d}(\mathrm{S})$, the drift, is often considered as degradation rate and is often used as an accelerated model; $\sigma$ is a constant and $\sigma>0 ; \mathrm{B}(\mathrm{t})$ is a standard Brownian motion on $[0, \infty)$, $\mathrm{B}(\mathrm{t}) \sim \mathrm{N}(0, \mathrm{t})$.

\subsection{Accelerated model}

The input electrical power of solid-state lasers is selected as stress variable in this study to conduct the SSADT. So the degradation rate $d(S)$ is supposed to follow Inverse Power Law model:

$$
d\left(S_{K}\right)=A S_{K}{ }^{B}
$$

Where, $\mathrm{S}_{\mathrm{k}}$ is the absolute input electrical power at $k$ th stress level, and A, B are positive constants.

\subsection{Reliability model}

Considering the unique feature of solid state lasers when choosing the input electrical power as step stress, the observation variable here is not the degradation variable, but the product of the degradation variable and accelerate stresses. The formula can be presented as follows:

$$
P=Y(t) \times S=\left(y_{0}-d(s) \cdot t+\sigma B(t)\right) \times S
$$

With a specified threshold value $C$, the product's lifetime $\mathrm{T}$ follows the Inverse-Gaussian distribution ${ }^{[10]}$ with the probability density function:

$$
f\left(t ; C, y_{0}\right)=\frac{y_{0}-C / \mathrm{S}}{\sigma \sqrt{2 \pi t^{3}}} \exp \left\{-\frac{\left[y_{0}-C / \mathrm{S}-d(S) \cdot t\right]^{2}}{2 \sigma^{2} t}\right\}
$$

Reliability function of solid state lasers can be obtained as:

$$
\begin{aligned}
& R(t)=\Phi\left[\frac{y_{0}-C / \mathrm{S}_{0}-d\left(\mathrm{~S}_{0}\right) \cdot t}{\sigma \sqrt{t}}\right] \\
& -\exp \left\{\frac{2 d\left(\mathrm{~S}_{0}\right) \times\left(y_{0}-C / \mathrm{S}_{0}\right)}{\sigma^{2}}\right\} \Phi\left[-\frac{y_{0}-C / \mathrm{S}_{0}+d\left(\mathrm{~S}_{0}\right) \cdot t}{\sigma \sqrt{t}}\right]
\end{aligned}
$$

\section{Parameter estimation}

In this section, the unknown parameters of the step stress accelerated degradation model are estimated by the maximum likelihood estimation method. Suppose all of the samples are under K-level SSADT, $\mathrm{S}_{1}<\mathrm{S}_{2}<\cdots<\mathrm{S}_{\mathrm{K}}$. Performance inspection interval of optical output power is $\Delta t$. There are $M_{k}$ times of performance examination in the process of SSADT, each of which is under the stress level $\mathrm{S}_{\mathrm{k}}$, besides, the accumulated inspections are $\mathrm{M} . \mathrm{P}_{\mathrm{ikj}}$ is the inspection result of the jth inspection on the ith sample under the $S_{k}$ stress level, let $\Delta D_{i k j}=P_{i k(j-1)}-P_{i k j}$, so 


$$
\Delta D_{i k j} \sim N\left(d\left(S_{k}\right) \Delta t S_{k}, \sigma^{2} \Delta t S_{k}^{2}\right)
$$

The likelihood function is:

$$
L=\prod_{i=1}^{n} \prod_{k=1}^{K} \prod_{j=1}^{M_{i}} \frac{1}{\sqrt{2 \pi \sigma^{2} \Delta t S_{k}{ }^{2}}} \exp \left\{-\frac{\left[\Delta D_{i k j}-A S^{B}{ }^{B} \Delta t S_{k}\right]^{2}}{2 \sigma^{2} \Delta t S_{k}{ }^{2}}\right\}
$$

Then, the log-likelihood formula is:

$$
\left.\ln L=-\frac{1}{2} \sum_{i=1}^{n} \sum_{k=1}^{K} \sum_{j=1}^{M_{k}}+\frac{\left(\Delta D_{i k j}-A S_{k}{ }^{B+1} \cdot \Delta t\right)^{2}}{\sigma^{2} \Delta t S_{k}{ }^{2}}\right\}
$$

Calculate the partial derivative of parameters A, B and $\sigma^{2}$ :

$$
\begin{aligned}
& \frac{\partial \ln L}{\partial \ln A}=\sum_{i=1}^{n} \sum_{k=1}^{K} \sum_{j=1}^{M_{i}} \frac{\Delta t S_{k}{ }^{B+1}\left(\Delta D_{i j}-A S_{k}{ }^{B+1} \Delta t\right)}{\sigma^{2} \Delta t S_{k}{ }^{2}} \\
& \frac{\partial \ln L}{\partial \ln B}=\sum_{i=1}^{n} \sum_{k=1}^{K} \sum_{j=1}^{M_{i}}\left\{\begin{array}{l}
\frac{A \cdot \Delta t \cdot \ln S_{k} \cdot \Delta D_{i k j} \cdot S_{k}^{B+1}}{\sigma^{2} \cdot \Delta t \cdot S_{k}{ }^{2}} \\
-\frac{A^{2} \cdot \Delta t^{2} \cdot \ln S_{k} \cdot\left(S_{k}{ }^{B+1}\right)^{2}}{\sigma^{2} \cdot \Delta t \cdot S_{k}{ }^{2}}
\end{array}\right\} \\
& \frac{\partial \ln L}{\partial \ln \sigma^{2}}=-\frac{1}{2} \sum_{i=1}^{n} \sum_{k=1}^{K} \sum_{j=1}^{M_{i}}\left\{\frac{2}{\sigma}-\frac{\left(\Delta D_{i k j}-A S_{k}{ }^{B+1} \Delta t\right)^{2}}{\sigma^{4} \cdot \Delta t \cdot S_{k}{ }^{2}}\right\}
\end{aligned}
$$

Let the above partial derivatives be zero, and the maximum likelihood estimation of $\mathrm{A}, \mathrm{B}$ and $\sigma^{2}$ can be calculated as follows:

$$
\begin{gathered}
\hat{A}=\sum_{i=1}^{n} \sum_{k=1}^{K} \sum_{j=1}^{M_{k}} \frac{\Delta D_{i j}}{\Delta t \cdot S_{k}{ }^{B+1}} \\
\hat{B}=\sum_{i=1}^{n} \sum_{k=1}^{K} \sum_{j=1}^{M_{k}}\left(\ln \frac{\Delta D_{i k j}}{A \cdot \Delta t}-1\right) \\
\hat{\sigma}^{2}=\sqrt[3]{\frac{4\left(\Delta D_{i k j}-A S^{B+1} \Delta t\right)^{4}}{\Delta t^{2} \cdot S_{k}{ }^{4}}}
\end{gathered}
$$

\section{Example}

Take a YAG solid state laser as the research object. The sample size is 6 and the 3 stress levels are $240 \mathrm{~W}, 280 \mathrm{~W}$ and $320 \mathrm{~W}$, respectively. Performance inspection interval of output power is once an hour and there are 200 measurements under each stress. The test results are shown in Figure 2.

It can be seen from the performance trends with time that performance changes show randomness and stochastic process model can be well used here.

According to formulas (13), (14) and (15), we can obtain $\hat{A}=1.057 \times 10^{-4}, \hat{B}=1.686 \times 10^{-2}, \hat{\sigma}^{2}=4.784 \times 10^{-3}$. Bring the parameter values into Equation (6) and assume that the normal operating input power is $200 \mathrm{~W}$ and failure threshold $\mathrm{C}$ is 40 , the reliability function curve can be shown in Figure 3.

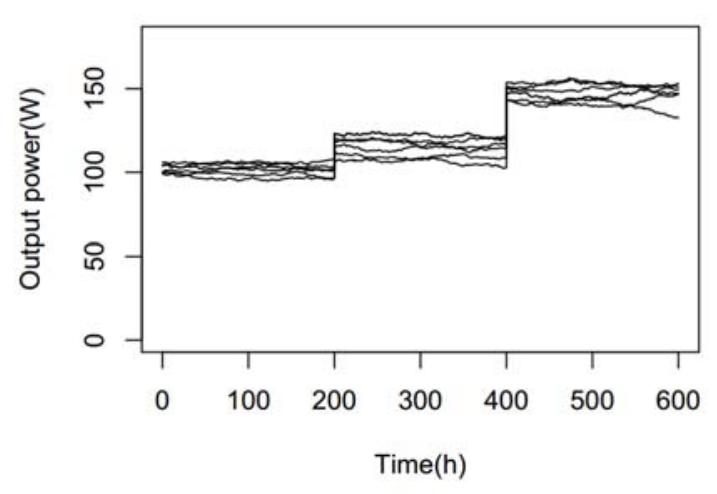

fig.2 Output power's variation of YAG solid state lasers

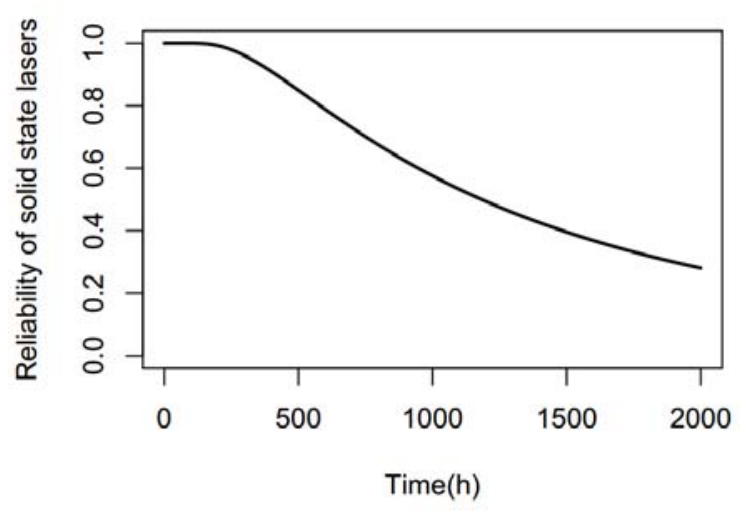

fig.3 Reliability curve of YAG solid state lasers

\section{Conclusions}

In this paper, we introduced a SSADT for solid state lasers to evaluate their life and reliability. Stochastic process was used to model the degradation path of photoelectric change efficiency. Based on the output characteristic and failure mode effect of solid state lasers, inverse power law model was chosen and reliability model of solid state lasers was established. And then the model parameters were estimated by means of maximum likelihood estimation. Finally, a set of YAG solid-state lasers data was used to illustrate the proposed method.

\section{References}

[1] Nelson, W. B. 1990. Accelerated testing: statistical models, test plans, and data analysis. New York: John Wiley \&Sons.

[2] Iuculano, G., \& Zanini, A. 1986. Evaluation of failure models through step-stress tests. IEEE Transactions on Reliability, 35(4), 409-413.

[3] CHEN Xun, ZHANG Chunhua.2009. Research, application and development of accelerated testing. Journal of Mechanical Engineering, 45(8): 30-136. 
[4] Meeker, W. Q. and Escobar, L. A. 1998. Statistical Methods for Reliability Data. John Wiley \& Sons.

[5] Chao, M. T. 1999. Degradation analysis and related topics: Some thoughts and a review. The Proceedings of the National Science Council, Part A. 23, 555-566.

[6] Tseng S T, Peng C Y. 2007. Stochastic diffusion modeling of degradation data. Journal of Data Science, 5(3):315-333.

[7] Wang, H., Wang, G. J., \& Duan, F. J. 2016. Planning of step-stress accelerated degradation test based on the inverse gaussian process. Reliability Engineering System Safety, 154, 97-105.

[8] Lu J C, Park J, Yang Q. 1997. Statistical Inference of a Time-to-Failure Distribution Derived From Linear Degradation Data [J]. Technometrics, 39(4):391-400.

[9] Zhao W, Elsayed E A. 2004. An accelerated life testing model involving performance degradation[C]// Reliability and Maintainability, 2004 Symposium Rams. IEEE, 324-329.

[10] Doksum, K. A., \& Hóyland, A. 1992. Models for variable-stress accelerated life testing experiments based on wiener processes and the inverse gaussian distribution. Technometrics, 34(1), 74-82. 